M.J. Kelly

\title{
Nanotechnology and manufacturability
}

ABSTRACT. Between a promising initial result in a laboratory and the commercial availability of a product there are many hoops to jump through, and indeed there is a severe attrition rate so that few results actually make it to products. In this article, I want to focus on the technical aspects of achieving a manufacturable prototype, but it must be remembered that commercial success is based on customers actually wanting to buy the product in the market place at the price on offer. I am particularly concerned about the issue of manufacturability precisely because is not the concern of the authors of the vast majority of papers that appear under the title of nanotechnology, and the subset in nanoelectronics. Indeed I think that the problem is so serious that there will be a well-deserved backlash against the whole of nanoscience if it is not rectified soon.

Nanotechnology Perceptions 7 (2011) 79-81

Nonsubscribers: purchase individual article 